\section{Níveis de atividade física e estágios de mudanca de comportamento de universitários da área de saúde}

\section{Level of physical activity and behavior change stages among undergraduate students of health sciences}

Ivo Eduardo Chanca Diniz de Souza

Ivan Luiz Padilha Bonfante 2

Frederico Bento de Moraes Junior ${ }^{3}$

Wendell Arthur Lopes

\section{RESUMO}

O presente estudo teve como objetivo verificar o nível de atividade física e estágios de mudança de comportamento em universitários da área de saúde. A amostra foi composta por 416 indivíduos, com idade de 20,6 \pm 2,8 anos, proveniente dos cursos de Educação Física $(n=67)$, Enfermagem ( $n=87)$, Farmácia $(n=79)$, Fisioterapia $(n=99)$ e Nutrição $(n=84)$. A massa corporal e a estatura foram reportadas e o índice de massa corporal (IMC) foi calculado pela equação de Quételet. Utilizou-se o Questionário Internacional de Atividade Física (IPAQ) - versão curta, para avaliar o nível de atividade física. Quanto ao estágio de mudança de comportamento, os indivíduos foram avaliados através do modelo transteorético de mudança de comportamento. Os resultados encontrados neste estudo indicaram que quase $30 \%$ dos acadêmicos da área de saúde foram considerados fisicamente inativos ou insuficientemente ativos, sendo 6\% no curso de Educação Física, 34,5\% em Enfermagem, 35,5\% em Farmácia, 28,3 em Fisioterapia e 40,5 em Nutrição. Em relação ao estágio de mudança de comportamento, mais de $35 \%$ estavam se preparando para iniciar a prática de atividade física num futuro próximo. Conclui-se que, com exceção dos estudantes de Educação Física, há um número elevado de fisicamente inativos e insuficientemente ativos entre os acadêmicos dos cursos avaliados, mesmo se tratando de jovens e estudantes de cursos da área da saúde. Além disso, uma grande quantidade dos estudantes está propensa a mudar seu estilo de vida e terem uma vida mais ativa, o que deveria estimular o surgimento de mais oportunidades para a prática de atividade física.

\section{PALAVRAS-CHAVE}

Atividade motora; Estilo de vida; Estudantes.

\section{ABSTRACT}

This study aimed to verify the level of physical activity and behavior change stages among undergraduate students of health sciences. The sample was composed of 416 students majoring in Physical Education (n=67), Nursing ( $n=87)$, Pharmacy ( $n=79)$, Physiotherapy ( $n=99)$ and Nutrition $(n=84)$. Weight and height were assessed in order to calculate the body mass index (BMI). The International Physical Activity Questionnaire (IPAQ) was used to evaluate the level of physical activity. In relation to the behavior change stage, the individuals were evaluated through a transtheoretical model of health behavior change (TTM). The results found in this study indicate that almost $30 \%$ of the undergraduate students of health sciences are considered physically inactive or insufficiently active (6\% in Physical Education, 34,5\% in Nursing, 35,5\% in Pharmacy, 28,3\% in Physiotherapy, and $40,5 \%$ in Nutrition). In the stage of behavior change, more than $35 \%$ intend to engage in physical activity in the near future. We conclude that, with the exception of Physical Education students, there are high numbers of physically inactive and insufficiently active undergraduate students, especially when taking into consideration that the individuals are young and students at a university bealth sector. Moreover, there are also a lot of subjects likely to change and have a more active lifestyle, which should stimulate the emergence of more opportunities for physical activity.
Rev Bras Ativ Fís Saúde p. 608-617 DOI

http://dx.doi.org/10.12820/rbafs.v.20n6p608

1 Universidade Estadual do Centro-Oeste, Departamento de Educacão Física, Guarapuava, Paraná, Brasil

2 Universidade Estadual de Campinas, Faculdade de Educação Física, Campinas, São Paulo, Brasil 3 Universidade Federal do Paraná, Departamento de Educação Física, Curitiba, Paraná, Brasil

4 Universidade Estadual de Maringá, Departamento de Educação Física, Ivaiporã, Paraná, Brasil 


\section{INTRODUCCÃO}

Um estilo de vida fisicamente inativo está relacionado com o aumento substancial no desenvolvimento de doenças crônico-degenerativas como cardiopatias, hipertensão, diabetes, obesidade e alguns tipos de cânceres ${ }^{1}$. Estima-se que, em torno de $31 \%$ da população global, seja insuficientemente ativa ${ }^{2}$. No Brasil, dados referentes às capitais, mostraram que 49,4\% dos adultos são insuficientemente ativos e destes, $16,2 \%$ são considerados fisicamente inativos ${ }^{3}$.

Assim, o grande desafio dos profissionais da área da saúde têm sido desenvolver ações multidisciplinares que promovam a saúde, previnam doenças, modifiquem os comportamentos de risco e atendam interesses de bem-estar e qualidade de vida, a partir do incentivo a adoção de comportamentos saudáveis como, por exemplo, a prática regular de atividade física ${ }^{4}$.

Porém, nem mesmo os futuros profissionais da área da saúde, que têm em sua formação conceitos e conteúdos que tratam de comportamentos saudáveis, conseguem aliam isso a suas próprias vidas ${ }^{5,6}$. De fato, estudos que investigaram os níveis de atividade física em universitários de cursos da área da saúde em cidades da região sudeste ${ }^{7}$, centro-oeste ${ }^{8}$ e nordeste $^{9}$ do Brasil encontraram que, em torno de $22 \%$ a $35 \%$ dos universitários, eram fisicamente inativo ou insuficientemente ativos. $\mathrm{Na}$ região sul, nenhum estudo investigou os níveis de atividade física em acadêmicos da área da saúde, além dos cursos de Educação Física ${ }^{10,11}$ e Fisioterapia ${ }^{11}$.

Para uma intervenção efetiva com a atividade física, é importante ter conhecimento dos fatores que possibilitam as modificações de comportamento de uma população ${ }^{12}$. Identificar os estágios de mudança comportamental relacionados à atividade física pode ser considerado uma das primeiras etapas no processo de desenvolvimento de estratégias de intervenção, já que esforços específicos podem ser empregados para cada comportamento encontrado ${ }^{13}$.

Embora certos estudos no Brasil ${ }^{5-11,13-15}$ já tenham investigado os níveis de atividade física em universitários da área da saúde, não foram encontrados trabalhos que incluíram diferentes cursos da área da saúde na região sul do país. Além disso, são escassos os estudos que também incluíram a avaliação dos estágios para mudança de comportamento relacionado à atividade física em universitários ${ }^{10}$.

Baseado na importância da atividade física e do entendimento dos estágios de mudança de comportamento e, ainda, na lacuna de estudos com este objetivo em universitários de cursos da área da saúde na região sul do Brasil, o presente estudo buscou avaliar o nível de atividade física e os estágios de mudança de comportamento relacionado à atividade física em graduandos de diversos cursos da área da saúde de uma universidade pública do sul do Brasil.

\section{MÉTODOS}

\section{Sujeitos}

O presente estudo caracteriza-se como descritivo com delineamento transversal, em uma amostra representativa de acadêmicos de cursos da área da saúde, de uma universidade pública da região Centro-Oeste do Paraná, realizado no segundo semestre de 2011. A amostra foi calculada com base no número total de alunos matriculados no ano da coleta $(n=672)$ nos cinco cursos 
da área da saúde ofertada pela universidade (Educação Física, Enfermagem, Farmácia, Fisioterapia e Nutrição). Considerando um intervalo de confiança de $95 \%$, nível de precisão de $4 \%$ e proporção de adultos insuficientemente ativos na população brasileira de $50 \%^{3}$, a amostra mínima prevista foi de 317 acadêmicos. Para evitar uma possível perda amostral por recusa ou erros no preenchimento dos questionários, foram acrescidos $30 \%$ na amostra mínima, totalizando 413 acadêmicos.

A seleção amostral ocorreu por amostragem aleatória simples. Os sujeitos foram selecionados por sorteio, com base na relação de alunos regularmente matriculados nos cursos, utilizando o software Microsoft Excel@. Foram considerados elegíveis todos os alunos matriculados nos cursos da área da saúde. Os selecionados que não foram encontrados em sala, em três tentativas consecutivas, foram considerados perda amostral e os que não quiseram responder foram considerados recusas. Como critério de exclusão, considerou-se a impossibilidade de responder aos instrumentos por alguma condição física limitante.

Todos os participantes foram informados do objetivo da pesquisa e assinaram o Termo de Consentimento Livre e Esclarecido conforme projeto aprovado pelo Comitê de Ética em Pesquisa da UNICENTRO (Parecer nº 198/2010).

\section{Instrumentos}

Inicialmente, foi aplicado um questionário a fim de identificar as características da amostra (idade, sexo, curso e período) além de perguntas sobre as variáveis antropométricas (massa corporal e estatura). A partir da massa corporal e estatura reportados foi calculado o índice de massa corporal (IMC) pela divisão da massa corporal pela estatura ao quadrado.

O nível de atividade física foi avaliado por meio do Questionário Internacional de Atividade Física (IPAQ) - versão curta, o qual é validado para a população brasileira ${ }^{16}$. Para a estimativa dos níveis de atividade física foram consideradas a duração e a frequência semanal na forma de caminhada, atividades moderada e atividade vigorosa nos diferentes contextos da atividade física. Para a classificação dos níveis de atividade física com base na duração e frequência das atividades realizadas utilizou-se as seguintes categorias ${ }^{17}$ :

1) Muito ativo - aquele que ultrapassou as recomendações de atividades físicas: a) vigorosa: $\geq 5$ dias/sem e $\geq 30$ minutos por sessão; b) vigorosa: $\geq 3$ dias/sem e $\geq 20$ minutos por sessão + moderada e/ou caminhada: $\geq 5$ dias/ sem e $\geq 30$ minutos por sessão;

2) Ativo - aquele que cumpriu recomendações de atividades físicas: a) vigorosa: $\geq 3$ dias/sem e $\geq 20$ minutos por sessão; ou b) moderada ou caminhada: $\geq 5$ dias/sem e $\geq 30$ minutos por sessão; ou c) Qualquer atividade somada: $\geq 5$ dias/sem e $\geq 150$ minutos/sem (caminhada + moderada + vigorosa);

3) Insuficientemente ativo - aquele que realiza atividade física, porém insuficiente para ser classificado como ativo, pois não cumpre as recomendações quanto à freqüência ou duração. Para realizar essa classificação soma-se a freqüência e a duração dos diferentes tipos de atividades (caminhada + moderada + vigorosa). Este último grupo é dividido em dois subgrupos de acordo com os critérios de recomendação, porém no presente trabalho optamos por não realizar essa divisão a fim de melhor descrição dos resultados e há apenas o grupo de irregularmente ativos; 
4) Fisicamente inativo - aquele que não realizou nenhuma atividade física por pelo menos 10 minutos contínuos durante a semana.

Para detectar os estágios de mudança de comportamento relacionado à atividade física foi incluído também um questionário transteorético ${ }^{18} \mathrm{com}$ as seguintes opções de marcação:

1) Pré-contemplação - a qual o indivíduo não tem intenção de mudar o seu comportamento nos próximos seis meses;

2) Contemplação - o indivíduo tem a séria intenção de mudar o comportamento nos próximos seis meses;

3) Preparação - o indivíduo pretende agir num futuro próximo (em geral no próximo mês);

4) Ação - o comportamento já foi incorporado por menos de seis meses;

5) Manutenção - a ação já acontece há mais de seis meses e as chances de retorno ao antigo comportamento são mínimas.

Considerou-se como atividade física regular a prática de atividades físicas moderadas por, no mínimo 30 minutos, cinco ou mais dias por semana, ou, atividades físicas vigorosas por, no mínimo, 20 minutos, três ou mais dias na semana, ou ainda, qualquer atividade somada, por cinco ou mais dias na semana e 150 minutos ou mais por semana.

\section{Procedimentos}

Os questionários foram entregues aos acadêmicos, que receberam orientação sobre a maneira apropriada de preenchimento. As possíveis dúvidas sobre o correto preenchimento eram esclarecidas no mesmo momento pelos aplicadores dos questionários (acadêmicos do $4^{\circ}$ ano do curso de Educação Física treinados para essa finalidade).

\section{Análise estatística}

Para análise dos dados foi utilizada a estatística descritiva (média, desvio padrão, frequência absoluta e relativa). Para a comparação das variáveis contínuas (idade, massa corporal, estatura e IMC) foi utilizada a análise de variância (ANOVA one-way), seguido do teste de comparações múltiplas de Tukey, quando necessário. Para as comparações das variáveis nominais (nível de atividade física e estágio de mudança de comportamento) entre os cursos foi utilizado o teste Qui-Quadrado. Em todas as análises considerou-se como diferença estatística significativa o valor de $P \leq 0,05$.

\section{RESULTADOS}

A amostra final foi composta por 416 acadêmicos, sendo Educação Física ( $n=67)$, Enfermagem ( $n=87)$, Farmácia ( $n=79)$, Fisioterapia ( $n=99)$ e Nutrição $(n=84)$. A caracterização da amostra quanto à idade e variáveis antropométricas, divididos entre os cursos da área da saúde avaliados encontra-se na Tabela 1. A idade média dos universitários foi de 20,6 $\pm 2,8$ anos. Não foram encontradas diferenças estatisticamente significativas na idade e nas variáveis antropométricas entre os cursos. 
TABELA 1 - Média e desvio padrão (M DDP) das variáveis antropométricas dos estudantes avaliados, divididos entre os cursos.

\begin{tabular}{llccc}
\hline & $\mathrm{n}$ & Estatura $(\mathrm{m})$ & Peso $(\mathrm{kg})$ & $\mid \mathrm{MC}\left(\mathrm{kg} / \mathrm{m}^{2}\right)$ \\
\hline Ed. Física & 67 & $1,71 \pm 0,10$ & $66,2 \pm 13,60$ & $22,6 \pm 3,10$ \\
\hline Enfermagem & 87 & $1,70 \pm 0,07$ & $61,7 \pm 9,38$ & $22,1 \pm 2,80$ \\
\hline Farmácia & 79 & $1,70 \pm 0,08$ & $60,1 \pm 12,84$ & $21,3 \pm 3,26$ \\
\hline Fisioterapia & 99 & $1,70 \pm 0,09$ & $61,4 \pm 10,77$ & $22,1 \pm 2,93$ \\
\hline Nutrição & 84 & $1,60 \pm 0,06$ & $58,9 \pm 7,61$ & $21,7 \pm 2,23$ \\
\hline
\end{tabular}

IMC = índice de massa corporal.

As frequências percentuais nas diferentes categorias do nível de atividade física dos universitários investigados estão ilustradas na Figura 1. A maioria dos estudantes universitários foi considerada ativa $(53,6 \%)$, e em torno de $29,8 \%$ considerados insuficientemente ativos ou fisicamente inativos.

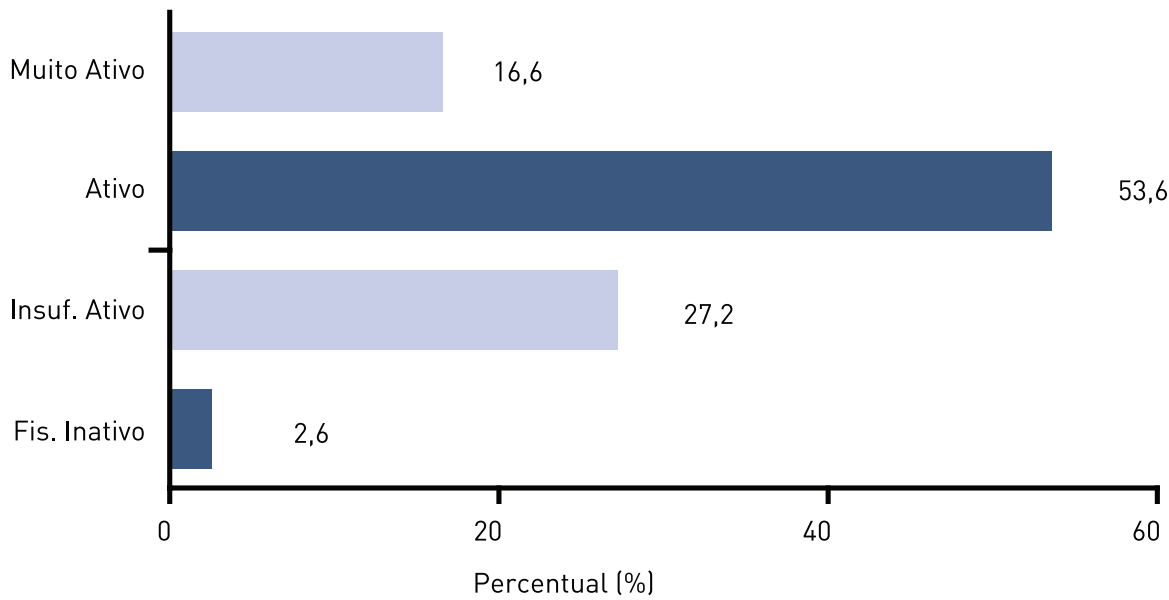

FIGURA 1 - Frequência percentual (\%) nas diferentes categorias do nível de atividade física de estudantes universitários da área da saúde.

$\mathrm{Na}$ Tabela 2, encontram-se os valores absolutos, relativos e intervalos de confiança (95\%) das diferentes categorias do nível de atividade física, divididos entre os cursos da área da saúde. O teste Qui-Quadrado revelou diferença estatística significativa na comparação das proporções dos níveis de atividades física entre os cursos $\left(x^{2}=47,264, d f=12, P<0,0001\right)$. Os acadêmicos do curso de Educação Física apresentaram valores percentuais significativamente menores de insuficientemente ativos $(4,5 \%)$ e maiores de muito ativos $(38,8 \%)$ em comparação aos outros cursos.

TABELA 2 - Valores absolutos e percentuais dos estudantes nos diferentes níveis de atividade física de acordo com o curso.

\begin{tabular}{lccccccccc}
\hline & Total & & Fis. Inativo & & Insuf. Ativo & \multicolumn{2}{c}{ Ativo } & \multicolumn{2}{c}{ Muito Ativo } \\
\hline & $\mathrm{n}$ & $\mathrm{n}$ & $\%\left(I \mathrm{C}_{95 \%}\right)$ & $\mathrm{n}$ & $\%\left(I \mathrm{C}_{95 \%}\right)$ & $\mathrm{n}$ & $\%\left(I \mathrm{C}_{95 \%}\right)$ & $\mathrm{n}$ & $\%\left(I \mathrm{C}_{95 \%}\right)$ \\
\hline Ed. Física & 67 & 1 & $1,5(0,0-7,9)$ & 3 & $4,5(1,5-12,4)^{*}$ & 37 & $55,2(43,3-66,5)$ & 26 & $38,8(28,0-50,8)^{*}$ \\
Enfermagem & 87 & 3 & $3,5(1,2-9,6)$ & 27 & $31,0(22,3-41,4)$ & 49 & $56,3(45,8-66,3)$ & 8 & $9,2(4,7-17,1)$ \\
\hline Farmácia & 79 & 2 & $2,5(0,7-8,8)$ & 26 & $33,0(23,5-43,8)$ & 37 & $46,8(36,2-57,7)$ & 14 & $17,7(10,9-27-6)$ \\
\hline Fisioterapia & 99 & 1 & $1,0(0,18-5,5)$ & 27 & $27,3(19,5-36,8)$ & 57 & $57,6(47,7-66,8)$ & 14 & $14,1(8,6-22,3)$ \\
\hline Nutrição & 84 & 4 & $4,8(1,9-11,6)$ & 30 & $35,7(26,3-46,4)$ & 43 & $51,2(40,7-61,6)$ & 7 & $8,3(4,1-16,2)$ \\
\hline
\end{tabular}

*Diferença significativa para os demais cursos. 
Em relação aos estágios de mudança de comportamento para a atividade física, a maioria dos estudantes $(42,5 \%)$ encontrou-se no estágio de manutenção e $36,1 \%$ no estágio de preparação. Não houve nenhum estudante no estágio de pré-contemplação (Figura 2).

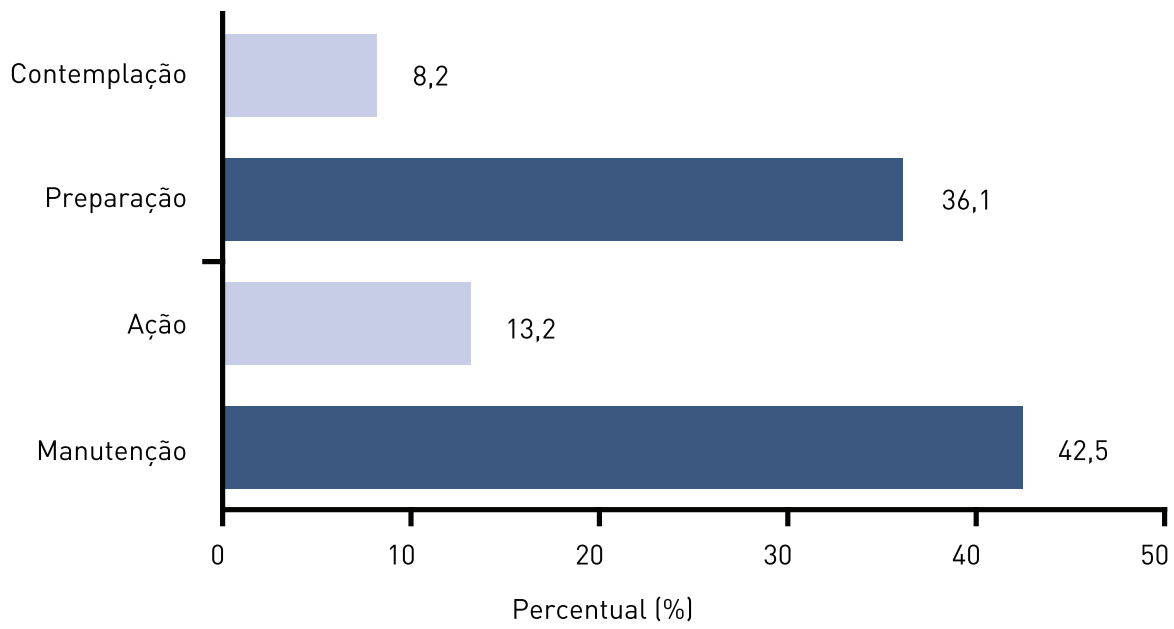

FIGURA 2 - Frequência percentual (\%) nos diferentes estágios de mudança de comportamento em relação à atividade física de estudantes universitários da área da saúde.

Os valores absolutos, relativos e intervalos de confiança (95\%) das diferentes categorias dos estágios de mudança de comportamento em relação à atividade física entre os diferentes cursos da área da saúde estão mostrados na Tabela 3. O teste Qui-Quadrado revelou diferença estatística significativa na comparação das proporções dos estágios de mudança de comportamento entre os cursos $\left(x^{2}=66,894, d f=12, P<0,0001\right)$. Os alunos do curso de Educação Física apresentaram valores percentuais significativamente menores nos estágios de contemplação $(0,0 \%)$ e preparação $(10,4 \%)$, além de maior percentual no estágio de manutenção $(82,1 \%)$ quando comparados com os alunos dos demais cursos.

TABELA 3 - Valores absolutos e percentuais dos estudantes nos diferentes estágios de mudança de comportamento relacionado à atividade física de acordo com o curso.

\begin{tabular}{|c|c|c|c|c|c|c|c|c|c|}
\hline & Total & & Contemplação & & Preparação & & Ação & & Manutenção \\
\hline & $n$ & $\mathrm{n}$ & $\%\left(\mid C_{95 \%}\right)$ & $\mathrm{n}$ & $\%\left(\mid C_{95 \%}\right)$ & $n$ & $\%\left(\mid C_{95 \%}\right)$ & $\mathrm{n}$ & $\%\left(\mid C_{95 \%}\right)$ \\
\hline Ed. Física & 67 & 0 & $0,0(0,0-5,4)^{*}$ & 7 & $10,4(5,1-20,0)^{*}$ & 5 & $7,5(3,2-16,3)$ & 55 & $82,1(71,2-89,4)^{*}$ \\
\hline Enfermagem & 87 & 11 & $12,7(7,2-21,2)$ & 39 & $44,8(34,8-55,3)$ & 8 & $9,2(4,7-17,1)$ & 29 & $33,3(24,3-43,8)$ \\
\hline Farmácia & 79 & 6 & $7,6(3,5-15,6)$ & 26 & $32,9(23,5-43,8)$ & 9 & $11,4(6,1-20,2)$ & 38 & $48,1(37,4-58,9)$ \\
\hline Fisioterapia & 99 & 8 & $8,1(4,1-15,1)$ & 39 & $39,4(30,3-49,2)$ & 21 & $21,2(14,3-30,3)$ & 31 & $31,3(23,0-41,0)$ \\
\hline Nutrição & 84 & 9 & $10,7(5,7-19,1)$ & 39 & $46,4(36,1-57,0)$ & 12 & $14,3(8,4-23,3)$ & 24 & $28,6(20,0-39,0)$ \\
\hline
\end{tabular}

*Diferença significativa para os demais cursos

\section{DISCUSSÃO}

O presente trabalho teve como objetivo avaliar o nível de atividade física e os estágios de comportamento relacionado à atividade física em universitários da área de saúde. Os resultados apontaram que 30\% dos universitários foram considerados fisicamente inativos ou insuficientemente ativos e $44,3 \%$ foram classificados nos estágios de contemplação ou preparação para mudança de comportamento em relação à atividade física. 
O número de universitários que não atingem um mínimo recomendável de atividade física no presente estudo é similar aos dados da população mundial de fisicamente inativos $(31,1 \%)^{2}$, bem como entre a faixa percentual encontrada em universitários de diferentes regiões do país $(21,8 \% \text { a } 34,5 \%)^{7-9}$.

Esses números são preocupantes já que atividade física é um fator primordial para uma boa saúde ${ }^{19}$ e ainda por se tratarem de jovens e futuros profissionais da área da saúde, dos quais se espera uma maior conscientização e multiplicação de hábitos saudáveis ${ }^{5,6}$. Rigoni et $\mathrm{al}^{15}$ apontam que as principais barreiras que levam universitários a se afastarem de um estilo de vida ativo são a extensa jornada de trabalho e estudo, a falta de companhia, os compromissos familiares, a falta de recursos financeiros, energia, equipamentos e espaços físicos, além da realização de tarefas domésticas.

A frequência percentual de fisicamente inativos e de ativos foi similar entre todos os cursos da área da saúde investigados. Porém, o curso de Educação Física apresentou menores valores percentuais de insuficientemente ativo $(4,5 \%)$ e maiores de muito ativo $(38,8 \%)$ em comparação aos outros cursos. O número de sujeitos ativos e muito ativos no curso de Educação Física totalizaram $94 \%$, valor esse bem acima dos percentuais encontrados nos outros cursos da saúde $(65,3 \%)$. Esse elevado percentual de ativos e muito ativos também foi encontrado por Silva et al. ${ }^{7}$, que encontraram $92 \%$ dos universitários do curso de Educação Física nessas duas categorias de atividade física. Guedes et al. ${ }^{10}$ também verificaram que quase $80 \%$ dos universitários do curso de Educação Física estavam nas categorias ativo e muito ativo. Porém, neste estudo, os autores avaliaram apenas universitários do curso de Educação Física.

Considerando que o curso de Educação física trata de aspectos relacionados à atividade física na sua prática profissional, era de se esperar que os níveis de atividade física fossem mais elevados que os outros cursos da área da saúde. Entretanto, é interessante notar que o maior número de indivíduos na categoria muito ativa foi o que realmente diferenciou o curso de Educação Física dos outros cursos. Provavelmente, os acadêmicos de Educação Física participam mais de atividades vigorosas ou moderadas na maioria dos dias da semana, seja nas atividades laborais ou no lazer, comparados aos acadêmicos de outros cursos da área da saúde. Se a maior frequência de acadêmicos do curso de Educação Física na categoria muito ativo estaria relacionada a melhores níveis de aptidão física ou de indicadores de saúde isso ainda não foi investigado.

Em relação ao percentual de fisicamente inativos e insuficientemente ativos entre os cursos da área da saúde, com exceção do curso de Educação Física (6,0\% fisicamente inativos + insuficientemente ativos), apresentaram valores entre 28,3 e 40,5\%. Esses resultados foram similares ao reportado em adultos na população geral ${ }^{3}$ e universitários da área da saúde ${ }^{7}$.

Embora tenhamos encontrado diferenças entre os níveis de atividade física de alunos de Educação Física quando comparados aos demais, os valores médios de IMC foram similares e dentro da faixa de normalidade em todos os cursos analisados. Futuros trabalhos poderiam analisar também a composição corporal conforme o nível de atividade física nesta população, a fim de verificar se diferenças nos níveis de atividade física poderiam influenciar na composição corporal de universitários.

Quanto aos estágios de mudanças de comportamento relacionado à atividade física, observou-se que a maioria dos sujeitos se encontrava no estágio de 
manutenção (42,5\%). Por outro lado, grande parte da amostra encontrava-se no estágio de preparação $(36,1 \%)$. É interessante notar que o curso de Educação Física apresentou maior número de sujeitos no estágio de manutenção $(82,1 \%)$ em comparação aos outros cursos. Quando analisamos os cursos da área da saúde sem o curso de Educação Física, observamos que, em média, aproximadamente $35 \%$ dos universitários estavam no estágio de manutenção, sendo a maioria (40,9\%) enquadrada no estágio de preparação.

Esses achados corroboram com outros estudos que verificaram uma maior proporção de acadêmicos do curso de Educação Física no estágio de manutenção $0^{8,13,15,20}$. Além disso, nossos achados em relação aos outros cursos da área da saúde (exceto Educação Física) foram similares ao encontrado por Madureira et $\mathrm{al}^{14}$ que verificaram que a maioria dos universitários (29 cursos incluídos) estava nos estágios de contemplação (32\%) e preparação (29,5\%). A falta de estudos que investigaram os estágios de mudança de comportamento em relação à atividade física em outros cursos da área da saúde, além da Educação Física impossibilitou comparações com nossos achados.

Quando analisamos os resultados obtidos por ambos os instrumentos utilizados, percebemos que não houve similaridade entre os níveis de atividade física e os estágios de mudança de comportamento relacionado à atividade física. No presente estudos, $29,8 \%$ dos sujeitos foram considerados fisicamente inativos ou insuficientemente ativos. Porém, 44,3\% foram classificados no estágio de contemplação ou preparação. Considerando que esses dois estágios pressupõem que o individuo não está praticando atividade física regularmente, era de se esperar valores similares em ambos. Sobre esse aspecto, Guedes et $\mathrm{al}^{10}$ verificaram uma incongruência entre a concepção dos estágios de mudança de comportamento e os níveis de atividade física aferidos pelo IPAQ entre acadêmicos de Educação Física, na qual os estágios de pré-adoção (contemplação e preparação) não são discriminadores adequados para identificar aqueles acadêmicos insuficientemente ativos. Os autores sugerem uma adequação no algoritmo para identificação dos estágios que inclua diferentes dimensões da prática de atividade física. Apesar dessa relação não ter sido um dos propósitos do presente estudo, a disparidade observado entre esses constructos pode estar relacionada a esta incongruência entre os instrumentos utilizados nos acadêmicos da área da saúde.

A quantidade significativa de acadêmicos da área da saúde que estão com perspectivas de mudança para uma vida mais ativa (36\%) deve ser algo a ser observado e motivo de ações estimuladoras para que mais indivíduos sejam fisicamente ativos, por meio do incentivo e disponibilização da prática de atividade física no meio universitário. Infelizmente, um aspecto que não foi verificado neste estudo foi a identificação de barreiras para a prática de atividade física, que são aspectos fundamentais a fim de ajudar nesse sentido e devem estar presentes em outros estudos relacionados ao tema.

Com exceção dos estudantes de Educação Física, a ideia de que universitários da área da saúde têm hábitos mais saudáveis em seu cotidiano, em função de terem acesso a fatores de educação em saúde, parece não ser totalmente correta, já que um número considerável de sujeitos não atende a recomendação mínima de atividade física para a manutenção de uma vida saudáve ${ }^{20}$. Esses achados corroboram com outros estudos que verificam hábitos de vida inadequados relacionados à alimentação desequilibrada e baixos níveis de atividade física em 
futuros profissionais da saúde, o que pode acarretar prejuízo a vida pessoal e talvez até a sua prática profissional de orientador e educador em saúde $5,7,15,16,20$.

O presente estudo apresenta diversas limitações, incluindo a avaliação dos níveis de atividade física nos diferentes domínios (lazer, deslocamento, trabalho), o uso de dados antropométricos auto-reportados e ainda o uso de apenas uma instituição de ensino superior para análise. Entretanto, o estudo abrangeu uma amostra representativa de universitários da área da saúde de universidade do sul do Brasil, e avaliou não apenas os níveis de atividade física, mas também os estágios para mudança de comportamento em relação a atividades física.

Em suma, os resultados do presente estudo mostraram que quase um terço dos estudantes da área da saúde são fisicamente inativos ou insuficientemente ativos e que cerca $44 \%$ estão nos estágios de contemplação e preparação. Entre os cursos, a Educação Física foi o que apresentou menores percentuais de insuficientemente ativos e maiores de muito ativos, além de menores percentuais nos estágios de contemplação/preparação e maiores no estágio de manutenção para mudança de comportamento em relação à atividade física. Esses dados reforçam a ideia que o tema atividade física seja mais abordado pelos cursos da área da saúde, assim como sejam mais estimuladas atividades extracurriculares ou de extensão que tenham a prática de atividade física como objetivo.

\section{Contribuição dos autores}

IECDS apresentou a ideia da investigação, realizou a coleta de dados e contribui na revisão de literatura. ILPB contribuiu na análise estatística, na redação do artigo, na elaboração final do manuscrito e revisão crítica do artigo. FBMJ contribuiu na redação do artigo e da revisão de literatura. WAL contribuiu na ideia da investigação, coleta de dados, na análise estatística, elaboração final e revisão crítica do artigo.

\section{Conflito de Interesses}

Declaramos não haver conflito de interesses pertinentes.

\section{Fontes de Financiamento}

$\mathrm{O}$ presente estudo não obteve financiamento por agência de fomento. $\mathrm{O}$ segundo e o último autor foram bolsistas de mestrado e de doutorado do $\mathrm{CNPq}$, respectivamente.

\section{REFERÊNCIAS}

1. Lee I, Shiroma EJ, Lobelo F, Puska P, Blair SN, Katzmarzyk PT. Effect of physical inactivity on major non-communicable diseases worldwide: an analysis of burden of disease and life expectancy. Lancet. 2012;380:219-29.

2. Hallal PC, Andersen LB, Bull FC, Guthold R, Haskell W, Ekelund U. Global physical activity levels: surveillance progress, pitfalls, and prospects. Lancet. 2012;380:247-57.

3. Brasil, Ministério da Saúde, Secretaria de Vigilância em Saúde. VIGITEL Brasil 2013: Vigilância de Fatores de Risco e Proteção para Doenças Crônicas por Inquérito Telefônico. 2014.

4. Souza SC, Loch MR. Intervenção do profissional de educação física nos núcleos de apoio à saúde da família em municípios do norte do Paraná. Rev Bras de Ativ Fís e Saúde. 2011;16 (1):5-10.

5. Paixão LA, Dias RMR, Prado WL. Estilo de vida e estado nutricional de universitários ingressantes em cursos da área de saúde do Recife/PE. Rev Bras de Ativ Fis e Saúde. 2010; 15(3):145-50. 
6. Silva DAS. Indicadores do estilo de vida e autoavaliação negativa de saúde em universitários de uma instituição pública do nordeste do Brasil. Rev Bras Ativ Fis e Saúde. 2012;17(4):263-69.

7. Silva GSF, Bergamaschine R, Rosa M, Melo C, Miranda R, Filho MB. Avaliação do nível de atividade física de estudantes de graduação das áreas saúde/biológica. Rev Bras Med Esporte. 2007;13(1):39-42.

8. Marcondelli P, Costa THM, Schmtz BAS. Nível de atividade física e hábitos alimentares de universitários do $3^{\circ}$ ao $5^{\circ}$ semestres da área da saúde. Rev Nutr. 2008;21(1):39-47.

9. Mendes Netto RS, Silva CS, Costa D, Raposo OFF. Nível de atividade física e qualidade de vida de estudantes universitários da área de saúde. Rev Bras de Ciênc da Saúde. 2012;10 (34):47-55.

10. Guedes DP, Santos CA, Lopes CC. Estágios de mudança de comportamento e prática habitual de atividade física em universitários. Rev Bras Cine Des Hum. 2006;8(4):5-15.

11. Amorim DA, Dias JA. Nível de Atividade Física de Estudantes de Educação Física e Fisioterapia da Universidade do Estado de Santa Catarina. Rev Digital. Buenos Aires. $2011 ; 15: 154$.

12. Petroski EL, Oliveira MM. Atividade Física de Lazer e Estágios de Mudança de Comportamento em Professores Universitários. Rev Port Cien Desp. 2008;8(2):209-18.

13. Silva DAS, Pereira IMM. Estágios de Mudança de comportamento para atividades físicas e fatores associados em acadêmicos de educação física. Rev Bras de Ativ Fís e Saúde. 2010;15 (1):15-20.

14. Madureira AS, Corseuil HX, Pelegrini A, Petroski EL. Associação entre estágios de mudança de comportamento relacionados à atividade física e estado nutricional em universitários. Cad de Saúde Pública. 2009;25(10):2139-46.

15. Rigoni PAG, Nascimento Junior JRA, Costa GNFS, Vieira LS. Estágios de mudança de comportamento e percepção de barreiras para a prática de atividade física em universitários do curso de Educação Física. Rev Bras Ativ Fis e Saúde. 2012;17(2):87-92.

16. Pardini R, Matsudo SMM, Araújo T, Matsudo VKR, Andrade E, Braggion G. Validação do Questionário Internacional de Nível de Atividade Física (IPAQ Versão 6): estudopiloto em adultos jovens brasileiros. Rev Bras Ciên e Mov. 2001;9: 45-51.

17. International Physical Activity Questionnaire (IPAQ). Guidelines for data processing and analysis of the International Physical Activity Questionnaire (IPAQ) - short and long forms. Disponível em: http://www.ipaq. ki.se/scoring.pdf. Acessado em 04 de outubro de 2015.

18. Plotnkoff RC, Hotz SB, Birkett NJ, Courneya KS. Exercise and the transtheoretical model: a longitudinal testo f a population sample. Prev. Med. 2001; 33: 441-52.

19. World Health Organization. Global recommendations on physical activity for health. Genebra: WHO; 2010. Disponível em: http://whqlibdoc.who.int/publications/ 2010/9789241599979_eng.pdf Acessado em outubro de 2015.

20. Cordeiro PC, Claumann GS, Pereira EF, Guimarães ACA, Pelegrini A. Estágios de mudança de comportamento relacionados à atividade física em acadêmicos de Educação Física. Rev Bras Ativ Fis e Saúde, 2014;19(4):484-486.

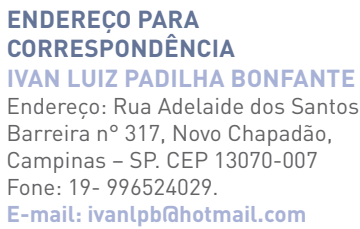

